\title{
Inducible and Constitutive Formation of $\beta$-Fructofuranosidase (Inulase) in Batch and Continuous Cultures of the Yeast Kluyveromyces fragilis
}

\author{
By J. W. D. GROOTWASSINK* AND G. M. HEWITT \\ Prairie Regional Laboratory, National Research Council, Saskatoon, Saskatchewan, \\ Canada S7N OW9
}

(Received 5 February 1982; revised 30 April 1982)

\begin{abstract}
Inulase production by Kluyveromyces fragilis on various fermentable and non-fermentable carbon sources was examined in carbon-limited continuous culture. Fructose and sucrose supported superior inulase yields [above $24 \mu \mathrm{mol}$ sucrose hydrolysed $\min ^{-1}(\mathrm{mg} \text { cell dry wt })^{-1}$ at $\mathrm{pH} 5.0,50^{\circ} \mathrm{C}$, while some other carbon sources, including lactose, galactose, ethanol and lactate, did not stimulate inulase formation beyond basal levels. Thus fructose was identified as the primary physiological inducer. Isolation of a constitutive mutant also provided genetic evidence for the inducible nature of inulase in the wild-type. The mutant was generated spontaneously and selected in continuous culture. It produced high inulase activities in continuous culture irrespective of the carbon source. Inulase formation in the wild-type and mutant strain was further controlled by general carbon catabolite repression as suggested by enzyme yield patterns in batch and continuous culture.
\end{abstract}

\section{INTRODUCTION}

The non-specific $\beta$-fructofuranosidase inulase is an external glycoprotein produced by a number of yeasts and filamentous fungi, among them Kluyveromyces (formerly Saccharomyces) fragilis (Snyder \& Phaff, 1960), Kluyveromyces lactis (Kovaleva \& Yurkevich, 1973), Kluyveromyces marxianus (Kierstan \& Bucke, 1977), Debaromyces cantarelli (Beluche et al., 1980), Candida kefyr (Negoro \& Kito, 1973), Aspergillus and Penicillium spp. (Kim, 1975; Nakamura et al., 1978). The yeast $K$. fragilis appears to give the highest enzyme yields (Kovaleva \& Yurkevich, 1973; Negoro, 1978; GrootWassink \& Fleming, 1980). Inulase rapidly splits terminal $\beta$-2,1- and $\beta$-2,6-fructofuranosidic bonds, which are present in sucrose, raffinose, stachyose and oligofructosides of the inulin or levan type (Snyder \& Phaff, 1960). This broad substrate spectrum renders the enzyme relatively non-specific in comparison with the other extensively studied, yeast $\beta$-fructofuranosidase, invertase. This latter enzyme acts rapidly on terminal $\beta$-fructofuranosidic bonds in disaccharides and trisaccharides but has an extremely low affinity (very high $K_{\mathrm{m}}$ ) for saccharides with a higher degree of polymerization (GrootWassink \& Fleming, 1980).

Since yeast cell membranes are impermeable to fructosides, including sucrose (Barnett, 1976; Sutton \& Lampen, 1962), the primary physiological function of inulase dictates an external location. The ratio of cell wall-bound inulase to soluble inulase depends on the cultivation conditions. High enzyme levels in yeast batch cultures have been observed only if the media contained oligofructosides such as inulin (Beluche et al., 1980; Negoro \& Kito, 1973; Snyder \& Phaff, 1960). However, experimental limitation of carbon catabolite repression by using the continuous culture technique has shown that the rapidly metabolizable substrate sucrose can also support high yields (GrootWassink \& Fleming, 1980).

In this paper we describe further efforts to identify environmental and genetic factors regulating inulase formation in $K$. fragilis. The range of culture conditions supporting enzyme production has been greatly expanded. 


\section{METHODS}

Organisms. The homothallic diploid yeast (Van Der Walt, 1970) Kluyveromyces fragilis ATCC 12424 (wild-type), and its spontaneous mutant strain PRL Y53A selected in continuous culture (see Fig. 4), were used. Genetic stability of stock cultures was safeguarded by preserving freshly grown vegetative cells of single-colony isolates in silica gel stored at $4^{\circ} \mathrm{C}$ (Fink, 1970). Inocula for experimental liquid cultures were grown by spreading a loopful of the silica gel mixture on an agar slant $\left(10 \mathrm{ml}\right.$ of $5 \mathrm{~g}$ yeast extract $\mathrm{l}^{-1}, 20 \mathrm{~g}$ glucose $1^{-1}$ and $20 \mathrm{~g}$ Bacto agar $\mathrm{l}^{-1}$ in a $25 \times 95 \mathrm{~mm}$ vial) and incubating for $3-4 \mathrm{~d}$ at $28^{\circ} \mathrm{C}$.

Liquid cultures. The media generally consisted of $5 \mathrm{~g}$ yeast extract $1^{-1}$ (Anheuser Busch, St Louis) to supply nitrogen, minerals and vitamins, plus one of the following carbon sources at a concentration of $10 \mathrm{~g}^{-1}:$ sucrose (Sigma, or technical grade beet sugar), glucose, fructose, mannose, galactose, lactose, inulin, glycerol, lactate, succinate, malate (all from Sigma) or ethanol (Fisher). In one experiment, the defined nutrients present in Yeast Nitrogen Base (Difco) were substituted for the complex yeast extract.

Batch cultures $(50 \mathrm{ml})$ were grown in $500 \mathrm{ml}$ Erlenmeyer flasks incubated at $28^{\circ} \mathrm{C}$ on a rotary shaker (280 r.p.m.). Continuous cultures were grown at $30^{\circ} \mathrm{C}$ in a 7.51 bench top fermenter (Microferm; New Brunswick Scientific). Sterile medium was pumped into the fermenter from 10 gallon (451) jugs or a medium sterilizer (Nutrient Vessel; New Brunswick Scientific). Spent culture and air were removed from the fermenter through a fixed overflow tube. Dissolved oxygen tension in continuous cultures was maintained around $20 \%$ of saturation by automatic adjustment of the impeller speed (300-500 r.p.m.) and aeration rate $\left(0.5-3.01 \mathrm{~min}^{-1}\right)$, employing a dissolved oxygen controller (model DO 81-14; New Brunswick Scientific). The pH of continuous cultures was maintained at 4.2 by automatic addition of $4 \mathrm{M}-\mathrm{HCl}$. Culture volumes varied with agitation and aeration rates and ranged from 2800 to $3000 \mathrm{ml}$. After experiments were completed at each dilution rate, the actual volumes were measured by interrupting agitation and aeration momentarily and reading a calibration scale on the fermentation vessel. Only minor fluctuations in agitation and aeration rates occurred during steady-state growth and had a practically negligible effect on the culture volume.

All media were inoculated with water-suspended cells from agar slant cultures at a ratio of one slant $1^{-1}$. Continuous cultivations were begun after initial batch growth in the fermenter had just reached the stationary phase as indicated by a rapid increase in dissolved oxygen tension. Cultures were grown at a fixed or sequentially increased dilution rate.

Analyses. Sets of shake flask cultures were sampled by randomly withdrawing flasks from the shaker. Continuous cultures were sampled by collecting $25-50 \mathrm{ml}$ spent culture in containers cooled in ice. Inulase activity was completely stable during sample collection and processing. To ensure that growth and enzyme biosynthesis had reached a steady-state in continuous culture, multiple sampling at 8-16 h intervals was performed after at least 3.5 culture volumes had passed through the fermenter at each dilution rate examined. For measuring inulase content, $0 \cdot 1-1 \mathrm{ml}$ samples of fresh whole culture samples were diluted 10-100 fold with acetate buffer (0.1 M, $\mathrm{pH} 5.0$ ) and kept frozen until assayed. For monitoring growth, samples were Millipore filtered (pore size $0.45 \mu \mathrm{m}$ ) and the dry weight of the yeast mat was determined after freeze-drying.

Inulase was assayed by incubating appropriately diluted whole culture samples $(0.1 \mathrm{ml})$ at $50{ }^{\circ} \mathrm{C}$ with $4 \%(\mathrm{w} / \mathrm{v})$ sucrose (Sigma) in $0.9 \mathrm{ml} 0.1 \mathrm{M}$-acetate buffer, pH 5.0 . Periodically, $0.1 \mathrm{ml}$ samples were withdrawn from the reaction mixture and analysed for an increase in total reducing sugars by the neocuproine method (Dygert $e$ al ., 1965). Thus, total inulase content could be measured since cell wall bound and soluble enzyme are both accessible to the substrate sucrose. Activity on inulin was occasionally verified but required a more elaborate assay procedure including cell disruption to minimize substrate diffusion limitations. The ratio of hydrolysis rates with $4 \%(w / v)$ sucrose and $4 \%(\mathrm{w} / \mathrm{v})$ inulin was $10 \cdot 5$, regardless of the source of inulase. Inulase activities are expressed as units $\mathrm{mg}^{-1}$, defined as $\mu \mathrm{mol}$ sucrose hydrolysed $\min ^{-1}$ ( $\mathrm{mg}$ cell dry $\left.\mathrm{wt}\right)^{-1}$.

\section{RESULTS}

\section{Continuous culture of the wild-type yeast}

Time course analysis of a batch culture of $K$. fragilis ATCC 12424 grown on inulin had previously shown a gradual increase in specific inulase activity until a maximum of 10 units $\mathrm{mg}^{-1}$ was reached at the end of the growth phase (GrootWassink \& Fleming, 1980). A more sudden appearance of inulase synthesis was observed when shake flask cultures were grown on glucose, fructose or sucrose : $80 \%$ of the total of only 2 units $\mathrm{mg}^{-1}$ was formed at the very end of the growth phase over a period of 2-4 h. Considering that growth in these cultures was carbonlimited, it appeared that low medium concentrations of carbon substrates, which prevailed at the end of the growth phase, were prerequisites for inulase synthesis. The higher initial concentrations of the carbon sources were probably causing catabolite repression. One way to limit this repression throughout the cultivation period is by use of the continuous culture 


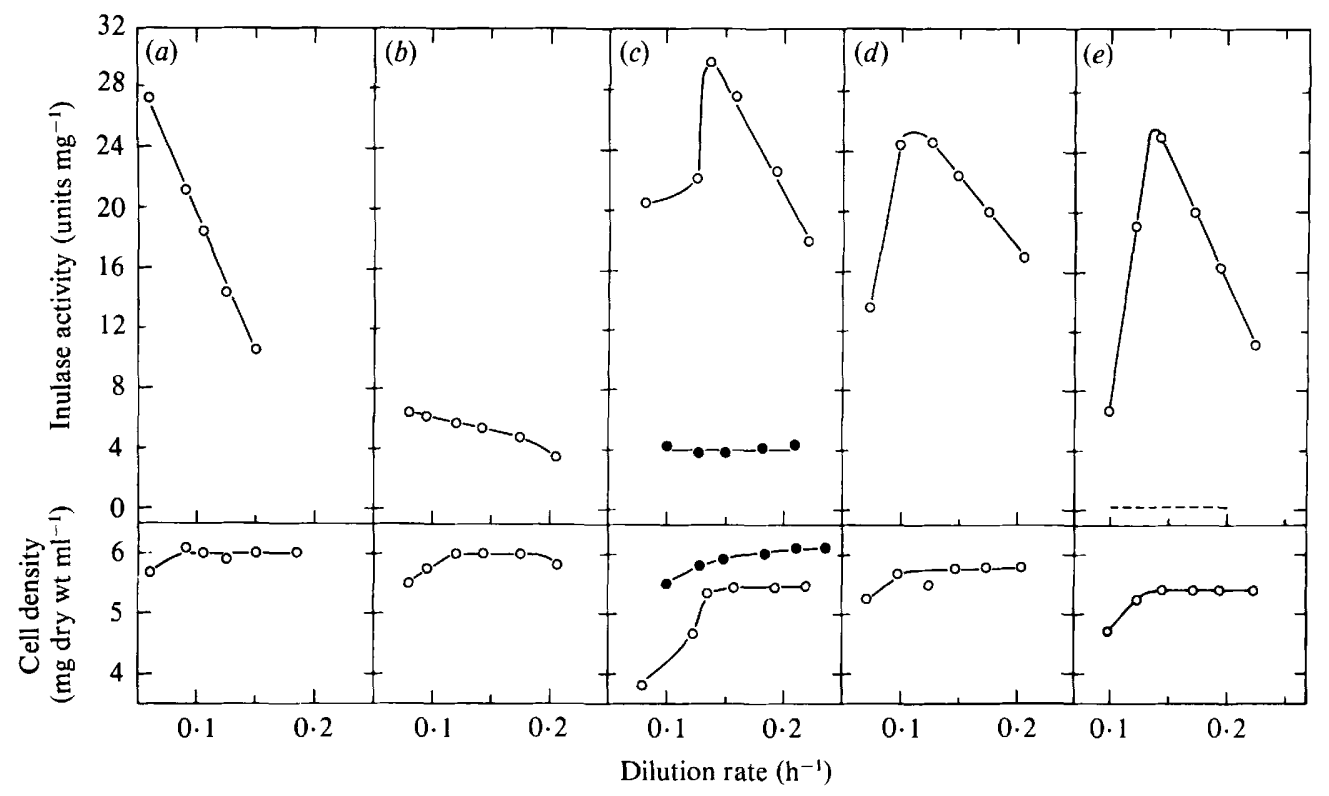

Fig. 1. Inulase activities in continuous cultures of $K$. fragilis wild-type grown on various carbon substrates. (a) Sucrose $(1 \%, w / v)$. (b) Glucose $(1 \%, w / v)$. (c) $\bigcirc$, Fructose $(1 \%, w / v) ; O$, mannose $(1 \%$, $w / v)$. (d) Fructose $(0.5 \%, w / v)$ plus glucose $(0.5 \%, w / v)$. (e) $\bigcirc$, Fructose $(0.5 \%, w / v)$ plus galactose $(0.5 \%, \mathrm{w} / \mathrm{v}) ;---$, lactose, galactose, ethanol or lactate (each at $1 \%, \mathrm{w} / \mathrm{v}$; results very similar).

technique. Here the growth-limiting nutrient can be maintained at very low but variable steadystate concentrations in the culture, depending on the dilution rate (Tempest, 1970). In the continuous cultures described in this paper the carbon sources were confirmed to be growthlimiting. That is, a small change in the chosen concentration of the carbon substrates, in otherwise unaltered feed media, caused a proportional change in the biomass yields. In addition, GLC analyses of a culture grown on sucrose (Fig. $1 a$ ) confirmed that very low sugar concentrations did indeed exist. The combined concentration of sucrose, glucose and fructose was around $20 \mathrm{mg}^{-1}$ at a dilution rate of $0.11 \mathrm{~h}^{-1}$.

Continuous cultures of the wild-type yeast were grown on a number of fermentable and nonfermentable carbon substrates over a range of dilution rates (Fig. 1). The most striking result from this substrate screening was that high inulase activities $\left(24\right.$ units $\mathrm{mg}^{-1}$ ) were produced on media containing fructose, present either bound in sucrose (Fig. $1 a$ ), alone (Fig. $1 c$ ) or in a mixture with other substrates (Fig. $1 d, e$ ). Glucose (Fig. $1 b$ ) and mannose (Fig. $1 c$ ) supported much lower activities ( 7 and 4 units $\mathrm{mg}^{-1}$, respectively), while lactose, galactose, ethanol and lactate gave basal activities of only $0 \cdot 1-0 \cdot 2$ units $\mathrm{mg}^{-1}$ (Fig. $1 e$ ). Maximum inulase activities were strongly dependent on the dilution rate. The sucrose-grown culture (Fig. 1 a) gave the highest activities at the lowest dilution rate $\left(0.06 \mathrm{~h}^{-1}\right)$. Cultures grown on free fructose showed a distinct inulase peak between 0.11 and $0.14 \mathrm{~h}^{-1}$ (Fig. $1 c, d, e$ ). These inulase profiles were reproducibly obtained under the standard operating conditions involving sequentially increased dilution rates. However, complications arose when a continuous culture, growing on fructose alone as carbon source, was passed through dilution rates in the reverse direction. The inulase yield rose dramatically to 42 units $\mathrm{mg}^{-1}$ as the dilution rate was decreased from $0.23 \mathrm{~h}^{-1}$ to $0.09 \mathrm{~h}^{-1}$ (Fig. 2).

To test the possibility that the complex nitrogen source yeast extract (which also provides small amounts of carbon) itself had a specific and major effect on inulase production, the defined and simple nitrogen source Yeast Nitrogen Base was used as a replacement in one experiment. It was complemented with glucose as the carbon source because of its intermediate capacity to support inulase. The results (Fig. 3) were similar to those presented earlier (Fig. $1 b$ ) for the 


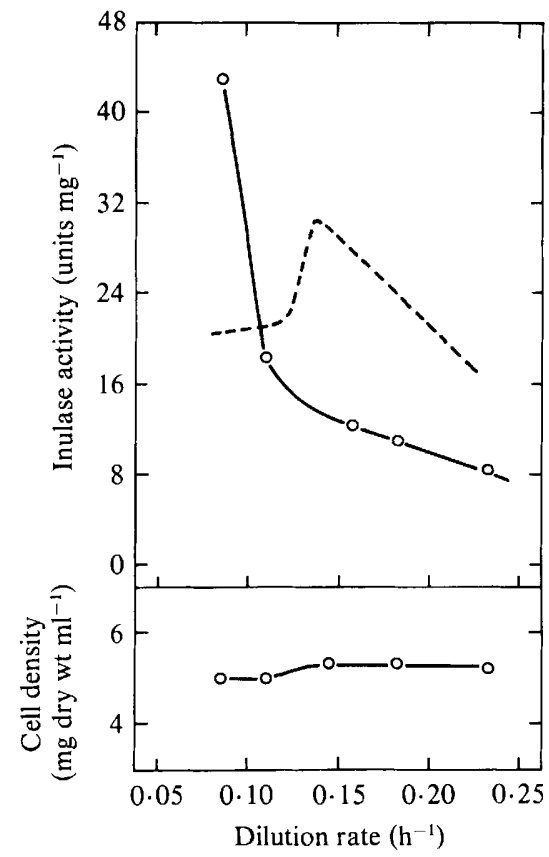

Fig. 2

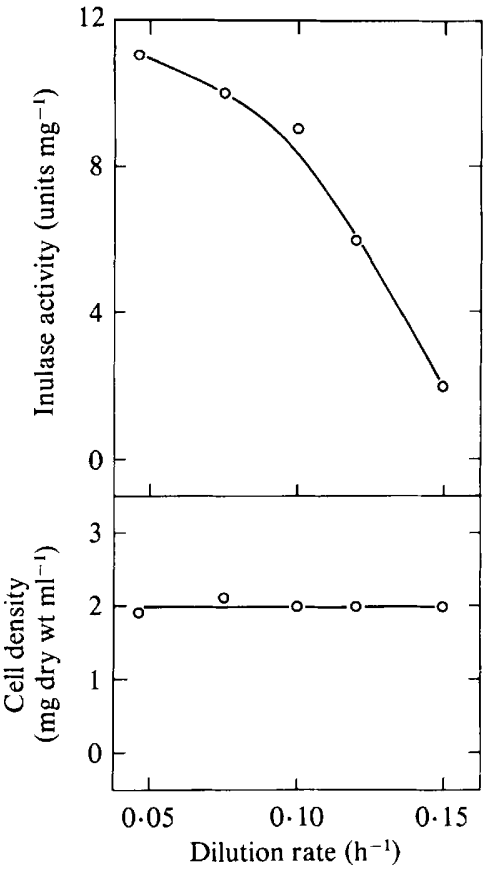

Fig. 3

Fig. 2. Inulase activities as a function of sequentially decreased dilution rates in a continuous culture of $K$. fragilis wild-type grown on fructose. -- Decreasing dilution rates; ---, increasing dilution rates (from Fig. 1c).

Fig. 3. Inulase activities in a continuous culture of $K$. fragilis wild-type grown on minimal medium containing $0.25 \%(\mathrm{w} / \mathrm{v})$ Yeast Nitrogen Base and $0.5 \%(\mathrm{w} / \mathrm{v})$ glucose.

culture grown on yeast extract. This indicated that the yeast extract used did not contain any substances overriding or interfering with the unique role of the primary carbon source in the regulation of inulase formation.

\section{Selection of constitutive mutants in continuous culture}

It was shown above that maximum inulase yields did not vary greatly if fructose alone (Fig. $1 c$ ) was replaced by equal mixtures of fructose and glucose (Fig. 1d) or fructose and galactose (Fig. $1 e$ ) as carbon sources. This finding suggested that perhaps only a minor proportion of the carbon source was needed in the form of fructose to effect high induction of enzyme synthesis. Thus, an experiment was done to test a range of fructose/galactose concentration ratios. Galactose was chosen as the supplementary carbon source because of its inability to stimulate inulase formation. The experiment was begun by first establishing high inulase levels in a culture growing on fructose alone at a dilution rate of $0 \cdot 14 \mathrm{~h}^{-1}$. To achieve this, the culture was passed through a few successively higher dilution rates beginning at $0.08 \mathrm{~h}^{-1}$ prior to fixing the rate at $0.14 \mathrm{~h}^{-1}$. Subsequently, the feed medium was changed at appropriate intervals to contain increasingly larger fractions of galactose (Fig. 4). The data show that a less than proportional drop in inulase occurred until a fructose/galactose concentration ratio of $3: 7$ was reached. Further replacement of fructose by galactose had the unexpected effect of causing a rise in the inulase yield until it reached 25 units $\mathrm{mg}^{-1}$ with galactose as the sole carbon source. The most attractive explanation of this phenomenon was that selection of a mutant population which no longer depended on fructose for inulase formation had taken place. To see whether this newly emerged population consisted of clones with a stably maintained constitutive phenotype, cells were plated and colonies picked for separate propagation on agar slants. The majority $(80 \%)$ had markedly improved inulase productivity over the wild-type strain in shake flask culture, suggesting that they were mutants. Continual propagation during 12 serial transfers of a 


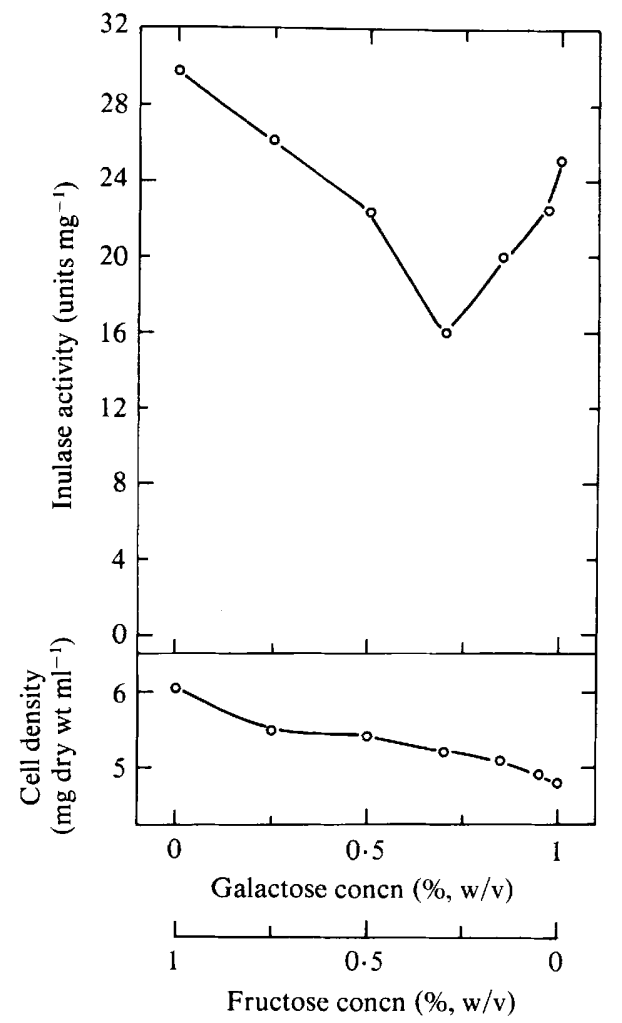

Fig. 4. Selection of an inulase-constitutive cell population in a continuous culture of $K$. fragilis. The culture was grown at a fixed dilution rate of $0.14 \mathrm{~h}^{-1}$. The composition of the carbon source in the feed medium was changed stepwise from $1 \%(w / v)$ fructose to $1 \%(w / v)$ galactose.

representative isolate, designated PRL Y53A, on agar slants did not result in any loss of the enhanced inulase productivity, indicating genetic stability of the new trait.

\section{Shake flask culture of an inulase-constitutive mutant}

A comparison of inulase yields in shake flask cultures of the wild-type and mutant PRL Y53A is presented in Table 1. Cultures were grown on fermentable and non-fermentable carbon sources. Without exception, all carbon sources supported much higher inulase activities in the mutant than in the wild-type. The increase ranged from 2-fold on inulin to 30 -fold on succinate. Maximum activities of 32 units $\mathrm{mg}^{-1}$ were obtained on the poor carbon source glycerol. In addition to testing for this overall effect on the final yield, two carbon sources, glucose and ethanol, were tested for their effect on the kinetics of inulase formation. As in the wild-type strain, the readily metabolizable substrate glucose completely repressed inulase synthesis in the mutant during exponential growth (Fig. 5). Exhaustion of glucose and limited post-exponential growth, presumably on secondary products and some components of the yeast extract, caused a rapid rise in inulase activity. The much more slowly metabolizable and non-fermentable carbon source ethanol supported enzyme formation during the entire growth phase, with activities reaching eight times higher values in the mutant (Fig. 6). However, some inulase repression was still apparent in these ethanol-grown cultures since the specific activity increased during the course of growth.

These results indicated that inulase synthesis in the mutant, as in the wild-type, is under the control of carbon catabolite repression. However, the second control mechanism, involving induction, appeared to be inoperative in the mutant. This conclusion was based on the finding that lactose, galactose, ethanol and lactate supported enormously higher activities (50-170 times higher) in the mutant grown in shake flask cultures, as compared to the wild-type grown in continuous culture on the same substrates under non-repressive conditions. 
Table 1. Inulase yields of shake flask cultures of $K$. fragilis wild-type and mutant PRL Y53A

The media contained yeast extract at $0.5 \%(\mathrm{w} / \mathrm{v})$ and carbon substrate at $1.0 \%(\mathrm{w} / \mathrm{v})$ except in the case of lactate, succinate and malate which were added at $0.5 \%(w / v)$ in combination with yeast extract at $0.25 \%(\mathrm{w} / \mathrm{v})$. Cells grown on carbohydrates were harvested at $24 \mathrm{~h}$; the remainder were harvested at $40 \mathrm{~h}$.

\begin{tabular}{lcc}
$\begin{array}{c}\text { Carbon } \\
\text { substrate }\end{array}$ & $\begin{array}{c}\text { Inulase activity } \\
\left(\text { units } \mathrm{mg}^{-1}\right)^{*}\end{array}$ \\
\cline { 2 - 3 } Inulin & 10 & 19 \\
Wucrose & 2 & 9 \\
Glucose & 2 & 7 \\
Fructose & 2 & 6 \\
Lactose & 1 & 11 \\
Galactose & 1 & 9 \\
Glycerol & 3 & 32 \\
Ethanol & 2 & 17 \\
Lactate & $0 \cdot 3$ & 5 \\
Succinate & 0.3 & 10 \\
Malate & $0 \cdot 4$ & 14
\end{tabular}

* Defined as $\mu \mathrm{mol}$ sucrose hydrolysed $\min ^{-1}$ (mg cell dry wt) ${ }^{-1}$.

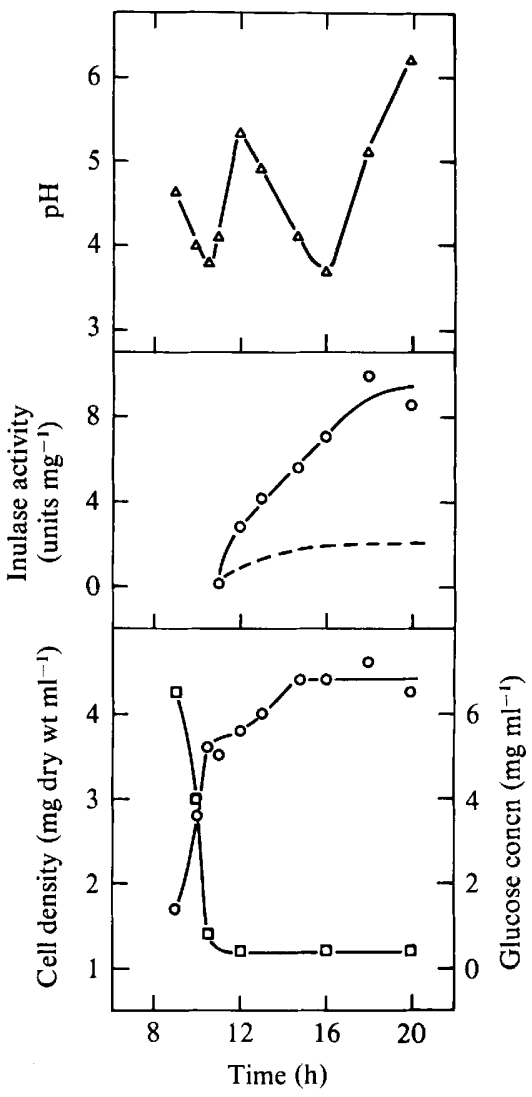

Fig. 5

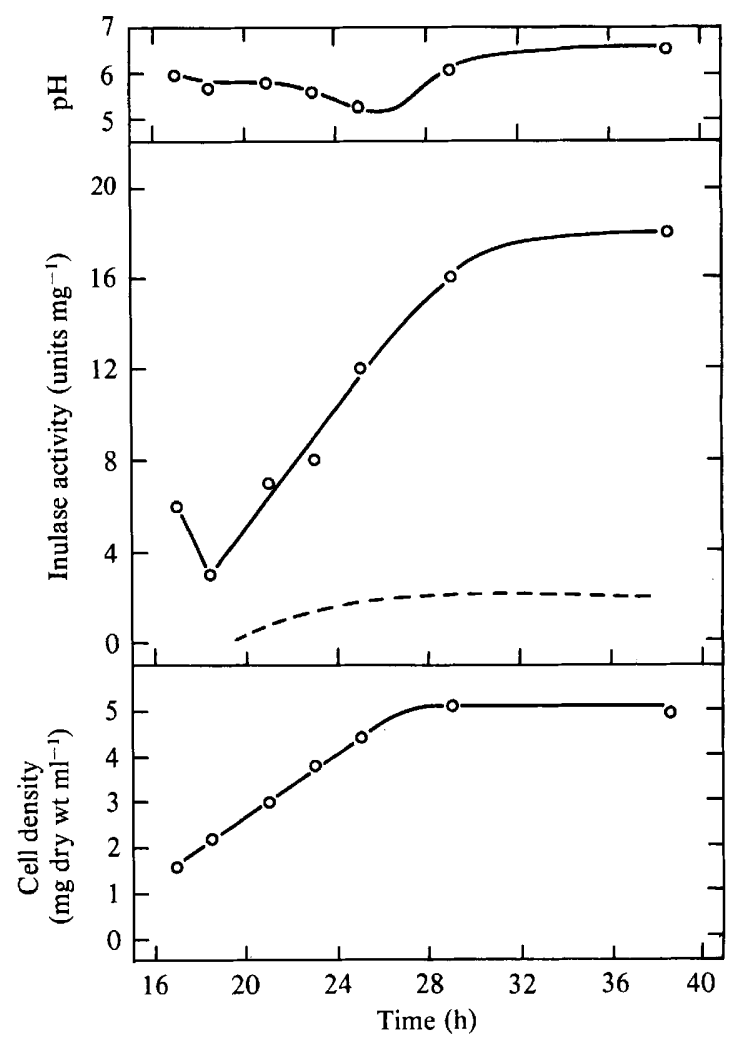

Fig 6

Fig. 5. Time course of shake flask cultures of a constitutive mutant of $K$. fragilis grown on glucose. Mutant PRL Y53A; ---, wild-type; $O$, cell density; $\square$, glucose concentration.

Fig. 6. Time course of shake flask cultures of a constitutive mutant of $K$. fragilis grown on ethanol. Mutant PRL Y53A; ---, wild-type. 


\section{Continuous culture of an inulase-constitutive mutant}

If indeed the hyperproducing mutant PRL Y53A was constitutive, it would be expected to produce high levels of inulase in continuous culture on all utilizable carbon sources. Special interest was focused on those substrates that had previously failed to give more than basal levels in continuous culture of the wild-type. The results in Table 2 show that enzyme yields of the mutant cultures grown on lactose, galactose, ethanol and lactate were similar to those obtained in wild-type cultures grown on the inducing substrates sucrose and fructose. The inulase levels were inversely related to the dilution rate, as exemplified in Fig. 7 for a mutant culture grown on galactose. The maximum activities observed were as high as 42 units $\mathrm{mg}^{-1}$ in a culture grown on glucose at the relatively low dilution rate of $0.08 \mathrm{~h}^{-1}$ (Table 2).

Table 2. Inulase yields of continuous cultures of $K$. fragilis wild-type and mutant PRL Y53A

$\begin{array}{lcccc}\begin{array}{c}\text { Carbon } \\ \text { substrate }\end{array} & \begin{array}{c}\text { Dilution } \\ \text { rate } \\ \left(\mathrm{h}^{-1}\right)\end{array} & \begin{array}{c}\text { Dry wt } \\ \left(\mathrm{mg} \mathrm{ml}^{-1}\right)\end{array} & \overbrace{\text { Wild-type }}^{\text {PRL Y53A }} \\ \text { Sucrose } & 0.11 & 6.0 & 18 & - \\ \text { Fructose } & 0.11 & 5.0 & 20 & - \\ \text { Glucose } & 0.08 & 5.0 & 7 & 42 \\ \text { Glucose } & 0.11 & 5.5 & 6 & 26 \\ \text { Lactose } \dagger & 0.17 & 5.6 & 0 \cdot 1 & 22 \\ \text { Galactose } & 0.11 & 5.4 & 0 \cdot 2 & 26 \\ \text { Ethanol } & 0.08 & 5.5 & 0.1 & 18 \\ \text { Lactate } & 0.11 & 4.2 & 0.1 & 22\end{array}$

* Defined as $\mu \mathrm{mol}$ sucrose hydrolysed $\min ^{-1}$ (mg cell dry wt) ${ }^{-1}$.

$\dagger$ Cells grown on lactose below a dilution rate of $0.14 \mathrm{~h}^{-1}$ showed an unstable constitutive phenotype.

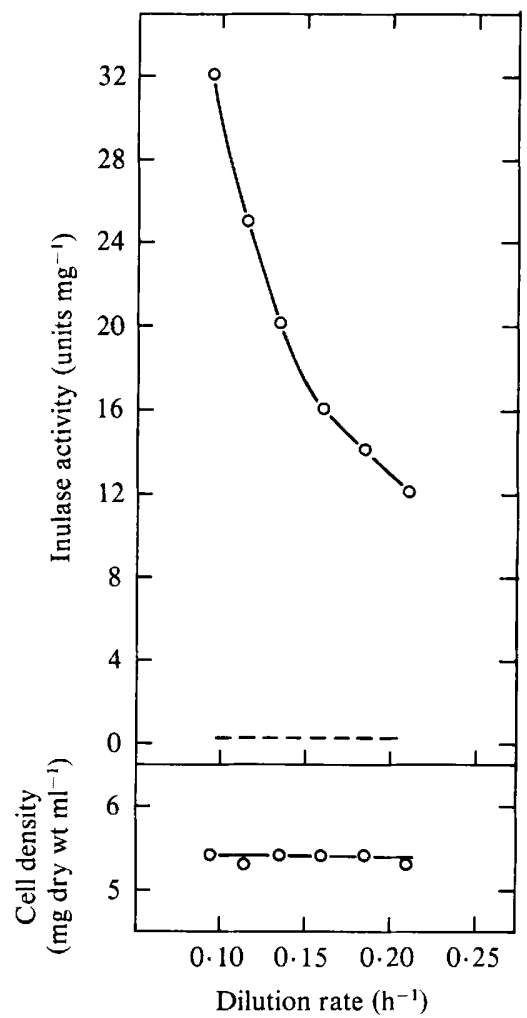

Fig. 7. Inulase production in a continuous culture of a constitutive mutant of $K$. fragilis grown on galactose. - Mutant PRL Y53A; - wild-type. 
The results clearly confirmed that the $K$. fragilis mutant does not require an inducer for inulase synthesis and thus is constitutive.

\section{DISCUSSION}

In micro-organisms, many catabolic enzymes with hydrolytic activity are subject to simultaneous induction and repression by a single growth substrate (Demain, 1971; Reese, 1972). Maximum enzyme expression depends on the optimum balance between the contribution of the two regulatory mechanisms. Both systems are strongly concentration dependent. Full induction of a gene requires saturation of a DNA-binding protein with a special inducer molecule, while minimum repression requires the lowest possible intracellular concentrations of certain substrate catabolites. A distinction must be made between a physiological inducer and a molecular inducer acting at the gene level. A physiological inducer might be the growth substrate itself or an enzymic break-down product thereof, as is the case with polymeric substrates. A molecular inducer is often a metabolic derivative of the physiological inducer. Non-metabolizable synthetic analogues may also be functional as molecular inducers. Only in very few instances have the natural molecular inducers been identified, the classical example being allolactose derived from lactose for $\beta$-galactosidase induction in Escherichia coli (Barkley et al., 1975). Cellulase induction in the mould Trichoderma reesei is believed to be mediated by sophorose derived from cellobiose which in turn is a product of cellulose hydrolysis (Sternberg \& Mandels, 1979).

The object of this work was to gain insight into the regulation of inulase formation by carbon substrates. The continuous culture technique was relied upon to provide much of the information. Profiles of several bacterial enzymes developed by determining the yields at different dilution rates had been interpreted by other workers to reflect the involvement of induction and/or repression mechanisms (Clarke et al., 1968; Dean, 1972; Bull, 1972). Similarly, in our study the peaking of inulase levels at intermediate dilution rates in cultures grown on media containing fructose could imply a balance between induction and repression (Fig. 1). Thus, a lack of inducer molecules predominated at the lower dilution rates and an excess of repressors at the higher rates. However, this interpretation became somewhat ambiguous after finding that inulase activities only peaked if the order of dilution rates was from low to high. In the converse order a totally different profile was observed, with the highest inulase yields produced at the lowest dilution rate (Fig. 2). Also contrary to expectations, when a culture which had passed through dilution rates from low to high was returned to a low rate, very high inulase activities $\left(\sim 40\right.$ units $\left.\mathrm{mg}^{-1}\right)$ were observed. This difference between the trajectory of ascent and descent is referred to as hysteresis and has previously been found to occur in continuous culture (Tanner, 1978). Its origin might lie in phenotypic and genotypic adaptations that vary with culture history. It is well known that continuous cultivation imposes tremendous selective pressures.

The rate of displacement of a cell population by a better adapted population is a function of the complex interaction between the rate of cellular changes (mutational and/or physiological) and increased specific growth rate. Every microbial continuous culture system has its unique selection characteristics, and no generalizations as to the time required for adaptation can be made. Although several genotypic adaptations in continuous cultures of bacteria are known (Harder et al., 1977), to date only a few such adaptations of yeasts have been reported. Downie \& Garland (1973) observed the emergence of a variant population of cells in about 48 generations ( $7 \mathrm{~d}$ ) in a copper-limited continuous culture of Candida utilis. The variants showed constitutive formation of a terminal oxidase alternative to cytochrome oxidase. In an ongoing study of the evolution of metabolic pathways, P. E. Hansche and co-workers are focusing on the genotypic adaptations of Saccharomyces cerevisiae in phosphate-limited continuous cultures. Mutations involving amino acid substitutions that effected a shift in the $\mathrm{pH}$ optimum and an increase in substrate affinity of acid phosphatase were observed after 180 generations (35 d) and 400 generations ( $77 \mathrm{~d}$ ), respectively (Francis \& Hansche, 1972). Mutants with increased acid phosphatase activities were isolated in another experiment after 400 generations, and were shown to result from gene duplication and transposition (Hansche, 1975; Hansche et al., 1978). 
After 1000 generations a cell population emerged with a mutation in a.repressor gene, resulting in constitutive formation of acid phosphatase (Lange \& Hansche, 1980). These examples show that it is difficult to affirm whether our cultures of $K$. fragilis on fructose lasted long enough (3040 generations) to allow genotypic adaptation. However, considering the clear-cut mutant selection that took place during only approximately 40 generations of growth on the mixed carbon substrates fructose and galactose (Fig. 4, discussed below), it cannot be ruled out that genotypic adaptation was also at least partially responsible for the observed hysteresis effect in the fructose-grown continuous cultures.

Other results which suggest that fructose may be the primary physiological inducer are the superior inulase yields on this carbon source in continuous culture. The fact that fructose is the breakdown product of inulase action on the natural oligomeric substrate inulin (Snyder \& Phaff, 1962) makes this monomer the obvious candidate. Thus, our experimental findings agree with this putative regulatory function of fructose. The next logical question is whether fructose itself is also the natural molecular inducer acting at the gene level. No direct answers may be expected from a physiological study like this. However, the observation that glucose and mannose also stimulate inulase formation could be explained in terms of a common glycolytic intermediate of the three monosaccharides, i.e. the actual gene inducer molecule. The marked differences between the inducing capacities of these sugars suggest that the pool size of the common intermediate is of critical importance. Mannose, which is catabolized like fructose except for a single isomerization step, gave only one-seventh as much inulase as fructose. Furthermore, galactose and lactose totally lacked induction capability, even though lactose contains a glucose moiety and both sugars presumably enter the glycolytic pathway after initial epimerization of the galactose.

The most convincing evidence for the broader conclusion that inulase is an inducible enzyme in wild-type $K$. fragilis comes from the isolation of a regulatory mutant which produced inulase constitutively. As long as catabolite repression was restricted, this mutant formed high levels of inulase regardless of the carbon source.

It is not obvious which forces were responsible for the selection of inulase constitutive mutants in the continuous culture in which fructose was gradually replaced by galactose as carbon substrate (Fig. 4). Development of mutant populations constitutive for substratecapturing enzymes has been noted in continuous cultures (Harder et al., 1977; Lange \& Hansche, 1980), but since inulase was not thought to have a fructose-capturing function, the present selection was totally unexpected. If inulase indeed has an affinity for fructose, mutant cells that contain large amounts of enzyme in the cell wall may have an advantage due to the higher fructose concentration in the micro-environment just outside the cell membrane. However, binding of frucrose would probably cause competitive inhibition of enzymic activity in vitro. This has neither been reported nor observed in our studies. Another explanation for selection could be that enzymes or transport proteins involved in fructose metabolism are controlled by the same regulatory mechanism as inulase. Similar co-regulation of enzymes of galactose and melibiose utilization has been observed in Saccharomyces carlsbergensis (Kew \& Douglas, 1976). Thus, mutants with enhanced fructose metabolism would show gratuitous hyperproduction of inulase.

Finally, it is tempting to compare the regulation of inulase formation to that of the very closely related and extensively studied yeast $\beta$-fructofuranosidase, invertase. At present, this enzyme is widely considered to be non-inducible and only controlled by carbon catabolite repression (Lampen, 1971; Zimmerman \& Scheel, 1977; Hackel \& Khan, 1978). However, conclusive biochemical and genetic evidence seems to be scanty. The conclusion is based mainly on the repressive effects of glucose and sucrose observed in continuous culture experiments using $K$. fragilis and Saccharomyces cerevisiae (Davies, 1956; Gascon \& Ottolenghi, 1972). Similar effects were observed on addition of several other carbon compounds to resting-cell cultures (Dodyk \& Rothstein, 1964). In the light of the present study on inulase, the data in these reports do not unequivocally exclude the involvement of an induction mechanism in the control of invertase formation. Simultaneous induction and repression by metabolic derivatives of the growth substrates, or lack of induction capability by the added compounds in the respective studies, 
could account equally well for the results. Induction of invertase has been recognized by Toda (1976) in a more recent publication on invertase formation in continuous cultures of $S$. carlsbergensis. Also, Zimmerman \& Scheel (1977), in a genetic study of S. cerevisiae mutants resistant to carbon catabolite repression, could not explain all their results without noting that perhaps repression is not the only mechanism controlling invertase formation.

\section{REFERENCES}

Barkley, M. D., Riggs, A. D., Jobe, A. \& Bourgeois, S. (1975). Interaction of effecting ligands with lac repressor and repressor-operator complexes. Biochemistry 14, 1700-1712.

BARNETT, J. A. (1976). The utilization of sugars by yeasts. Advances in Carbohydrate Chemistry and Biochemistry 32, 125-234.

Beluche, I., Guiraud, J. P. \& Galzy, P. (1980). Inulinase activity of Debaromyces cantarellii. Folia microbiologica 25, 32-39.

Bull, A. T. (1972). Environmental factors influencing the synthesis and excretion of exocellular macromolecules. Journal of Applied Chemistry and Biotechnology 22, 261-292.

Clarke , P. H., Houldsworth, M. A. \& Lilly, M. D. (1968). Catabolite repression and the induction of amidase synthesis by Pseudomonas aeruginosa 8602 in continuous culture. Journal of General Microbiology 51, 225-234.

DAVIES, A. (1956). Invertase formation in Saccharomyces fragilis. Journal of General Microbiology 14, 109-121.

DEAN, A. C. R. (1972). Influence of environment on the control of enzyme synthesis. Journal of Applied Chemistry and Biotechnology 22, 245-259.

Demain, A. L. (1971). Increasing enzyme production by genetic and environmental manipulations. Methods in Enzymology 22, 86-95.

DoDYK, F. \& RothsteIN, A. (1964). Factors influencing the appearance of invertase in Saccharomyces cerevisiae. Archives of Biochemistry and Biophysics 104, 478-486.

Downie, J. A. \& Garland, P. B. (1973). An antimycin A- and cyanide-resistant variant of Candida utilis arising during copper-limited growth. Biochemical Journal 134, 1051-1061.

Dygert, S., Li, L. H., Florida, D. \& Thoma, J. A. (1965). Determination of reducing sugar with improved precision. Analytical Biochemistry 13, 367374.

FINK, G. R. (1970). The biochemical genetics of yeast. Methods in Enzymology 17A, 59-78.

Francis, J. C. \& Hansche, P. E. (1972). Directed evolution of metabolic pathways in microbial populations. I. Modification of the acid phosphatase $\mathrm{pH}$ optimum in S. cerevisiae. Genetics 70, 59-73.

Gascon, S. \& OtTolenghi, P. (1972). Influence of glucose concentration of the medium on the invertase content of a strain of Saccharomyces bearing the SUC2 gene. Comptes rendus des travaux du Laboratoire Carlsberg 39, 15-24.

GrootWassink, J. W. D. \& Fleming, S. E. (1980). Non-specific $\beta$-fructofuranosidase (inulase) from Kluyveromyces fragilis: batch and continuous fermentation, simple recovery method and some industrial properties. Enzyme and Microbial Technology 2, 45-53.
HaCkel, R. A. \& KhaN, N. A. (1978). Genetic control of invertase formation in Saccharomyces cerevisiae. II. Molecular and General Genetics 164, 295-302.

HANSCHE, P. E. (1975). Gene duplication as a mechanism of genetic adaptation in Saccharomyces cerevisiae. Genetics 79, 661-674.

Hansche, P. E., Beres, V. \& Lange, P. (1978). Gene duplication in Saccharomyces cerevisiae. Genetics 88 , 673-687.

Harder, W., Kuenen, J. G. \& Matin, A. (1977). Microbial selection in continuous culture. Journal of Applied Bacteriology 43, 1-24.

Kew, O. M. \& Douglas, H. C. (1976). Genetic coregulation of galactose and melibiose utilization in Saccharomyces. Journal of Bacteriology 125, 3341.

Kierstan, M. \& BuCKe, C. (1977). The immobilization of microbial cells, subcellular organelles, and enzymes in calcium alginate gels. Biotechnology and Bioengineering 19, 387-397.

KIM, K. C. (1975). Studies on the hydrolysis of inulin in Jerusalem artichoke by fungal inulase. Journal of the Korean Agricultural and Chemical Society 18, 177182.

Kovaleva, N. S. \& YuRkevich, V. V. (1973). Sucrose and inulin hydrolysis by $\beta$-fructosidase from Saccharomyces (Kluyveromyces) lactis. Vestnik Moskovskogo gosudarstvennogo universiteta. Biologiya, Pochvovedenie 28, 37-43. (Translated by the National Research Council of Canada.)

LAMPEN, J. O. (1971). Yeast and Neurospora invertases. In The Enzymes, 3rd edn, vol. 5, pp. 291-305. Edited by P. D. Boyer. New York \& London: Academic Press.

LANGe, P. \& Hansche, P. E. (1980). Mapping of a centromere-linked gene responsible for constitutive synthesis in yeast. Molecular and General Genetics 180, 605-607.

Nakamura, T., Hoashi, S. \& Nakatsu, S. (1978). Culture conditions for inulase production by Aspergillus. Nippon Kogeikagaku Kaishi 52, 105-110.

NEGoRo, H. (1978). Inulase from Kluyveromyces fragilis. Journal of Fermentation Technology 56, 102107.

Negoro, H. \& KiTo, E. (1973). $\beta$-Fructofuranosidase from Candida kefyr. Journal of Fermentation Technology 51, 96-102.

REESE, E. T. (1972). Enzyme production from insoluble substrates. Biotechnology \& Bioengineering Symposium no. 3, 43-62.

SNYDER, H. E. \& Phaff, H. J. (1960). Studies on a $\beta$ fructosidase (inulinase) produced by Saccharomyces fragilis. Antonie van Leeuwenhoek 26, 433-452.

SNyder, H. E. \& PhafF, H. J. (1962). The pattern of action of inulinase from Saccharomyces fragilis on inulin. Journal of Biological Chemistry 237, 24382442. 
SternberG, D. \& Mandels, G. R. (1979). Induction of cellulolytic enzymes in Trichoderma reesei by sophorose. Journal of Bacteriology 139, 761-769.

SUTTON, D. D. \& LAMPEN, J. O. (1962). Localization of sucrose and maltose fermenting systems in Saccharomyces cerevisiae. Biochimica et biophysica acta 56, 303-312.

TANNER, R. (1978). Kinetic hysteresis in enzyme and fermentation systems. Annual Reports on Fermentation Processes 2, 73-89.

TEMPEST, D. W. (1970). The continuous cultivation of microorganisms: 1. Theory of the Chemostat. Methods in Microbiology 2, 259-276.
TODA, K. (1976). Invertase biosynthesis by Saccharomyces carlsbergensis in batch and continuous cultures. Biotechnology and Bioengineering 18, 11031115.

WALT, J. P. VAN DER (1970). Criteria and methods used in classification. In The Yeasts: A Taxonomic Study, 2nd edn, pp. 34-113. Edited by J. Lodder. Amsterdam \& London: North Holland Publishing Co.

Zimmerman, F. K. \& ScheEl, I. (1977). Mutants of Saccharomyces cerevisiae resistant to carbon catabolite repression. Molecular and General Genetics 154, $75-82$. 